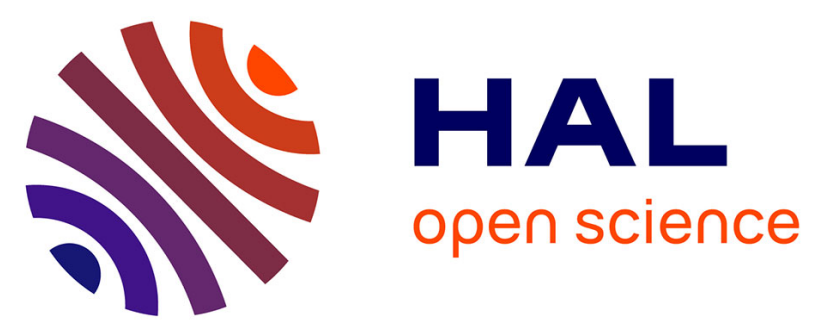

\title{
SIMULTANEOUS DETERMINATION OF THE MAGNETIC FIELD AND THE ELECTRIC POTENTIAL IN THE SCHRÖDINGER EQUATION BY A FINITE NUMBER OF BOUNDARY OBSERVATIONS
}

Ibtissem Ben Aicha, Youssef Mejri

\section{To cite this version:}

Ibtissem Ben Aicha, Youssef Mejri. SIMULTANEOUS DETERMINATION OF THE MAGNETIC FIELD AND THE ELECTRIC POTENTIAL IN THE SCHRÖDINGER EQUATION BY A FINITE NUMBER OF BOUNDARY OBSERVATIONS. 2016. hal-01351217

\author{
HAL Id: hal-01351217 \\ https://hal.science/hal-01351217
}

Preprint submitted on 2 Aug 2016

HAL is a multi-disciplinary open access archive for the deposit and dissemination of scientific research documents, whether they are published or not. The documents may come from teaching and research institutions in France or abroad, or from public or private research centers.
L'archive ouverte pluridisciplinaire HAL, est destinée au dépôt et à la diffusion de documents scientifiques de niveau recherche, publiés ou non, émanant des établissements d'enseignement et de recherche français ou étrangers, des laboratoires publics ou privés. 


\title{
SIMULTANEOUS DETERMINATION OF THE MAGNETIC FIELD AND THE ELECTRIC POTENTIAL IN THE SCHRÖDINGER EQUATION BY A FINITE NUMBER OF BOUNDARY OBSERVATIONS
}

\author{
IBTISSEM BEN AÏCHA, YOUSSEF MEJRI
}

\begin{abstract}
We study the inverse problem of determining the magnetic field and the electric potential appearing in the magnetic Schrödinger equation, from the knowledge of a finite number of lateral observations of the solution. We prove a Lipschitz stability estimate for both coefficients simultaneasly by choosing the "initial" conditions suitably.

Keywords: Inverse problem, magnetic Schrödinger equation, stability estimate, finite number of partial Neuman measurements, Carleman estimate.
\end{abstract}

\section{INTRODUCTION}

1.1. Statement of the problem. In this paper, we study the following inverse problem: Given $T>0$ and a bounded domain $\Omega \subset \mathbb{R}^{n}, n \geqslant 1$, with smooth boundary $\Gamma$, we want to determine simultaneously the divergence free magnetic potential of the form $a(x, t):=\chi(t) a(x)$ and the electric potential $q(x, t):=$ $\beta(t) q(x)$ appearing in the following equation

$$
\begin{cases}\left(-i \partial_{t}+H_{a}(t)+q(x, t)\right) u(x, t)=0, & \text { in } Q=\Omega \times(0, T), \\ u\left(x, \frac{T}{2}\right)=u_{0}(x) & \text { in } \Omega, \\ u(x, t)=0, & \text { on } \Sigma=\Gamma \times(0, T)\end{cases}
$$

where $H_{a}(t):=(i \nabla+\chi(t) a)^{2}$ denotes the time-dependent Hamiltonian, associated to the magnetic potential vector $\chi(t) a(x)$. Here $a=\left(a_{1}, \ldots, a_{n}\right) \in A:=H^{1}(\Omega)^{n} \cap\left\{a \in L^{\infty}\left(\Omega, \mathbb{R}^{n}\right), \quad \nabla \cdot a=0\right\}$, and $q \in L^{\infty}(\Omega)$ are unknown real valued functions. Moreover, the functions $\beta, \chi \in \mathcal{C}^{3}(0, T ; \mathbb{R})$ are assumed to be known functions satisfying

$$
\chi\left(\frac{T}{2}\right)=\beta\left(\frac{T}{2}\right)=0, \chi^{\prime}\left(\frac{T}{2}\right) \neq 0, \beta^{\prime}\left(\frac{T}{2}\right) \neq 0 .
$$

We denote by $\Gamma^{+}$an open subset of $\Gamma$ satisfying an appropriate geometrical condition we shall precise later.

The inverse problem we investigate in this paper, is to know whether the knowledge of a finite number of Neumann measurements $\partial_{\nu} u_{\mid \Gamma^{+} \times(0, T)}:=(\nabla u \cdot \nu)_{\mid \Gamma^{+} \times(0, T)}$ uniquely determines $a(x)$ and $q(x)$ simultaneously. Here $\nu(x)$ denotes the unit outward normal to $\Gamma$ at $x$.

1.2. Bibliographical notes. There is a wide mathematical literature dealing with uniqueness and stability in inverse coefficient problems related to partial differential equations. In recent years, significant progress have been made in the recovery of magnetic potentials in magnetic Schrödinger equations from the Dirichletto Neuman map. As it was noted in [11], the Dirichlet-to Neuman map is invariant under a gauge transfromation of the magnetic potential. Namely, given $\phi \in \mathcal{C}^{1}(\bar{\Omega})$ such that $\phi_{\mid \Gamma}=0$, we have $\Lambda_{a+\nabla \phi}=\Lambda_{a}$. So, due to this obstruction to uniqueness, the best one could recover from the Dirichlet-to-Neumann map is the magnetic field $d a$, where $d a$ is the exterior derivative of $a$ interpreted as the one form $\sum_{j=1}^{n} a_{j} d x_{j}$. 
The inverse problem of determining the magnetic field $d a$ and the electric potential $q$ from boundary observations was first considered by Sun [12], in the case $n \geqslant 3$. He showed that $d a$ and $q$ can be uniquely determined when $a \in W^{2, \infty}, q \in L^{\infty}$ and $d a$ is small in the $L^{\infty}$ norm. In [10], a uniqueness result was proved for $C^{\infty}$ magnetic potentials. In [11], Eskin proved a uniqueness result for an inverse problem for the Schrödinger equation with electromagnetic potentials, modulo a gauge transform of the recovery of the potentials from the Dirichlet-to Neuman map. In a recent work, Bellassoued and Choulli [3] considered the problem of recovering the magnetic potential $d a$ from the Dirichlet-to-Neumann map associated to the Schrödinger equation and proved in dimension $n \geqslant 2$ a stability estimate of Hölder type.

In the Riemannian case, Bellassoued [2] proved recently a Hölder-type stability estimate in the recovery of the magnetic field $d a$ and the electric potential $q$ from the knowledge of the Dirichlet-to-Neumann map associated to the Schrödinger equation with zero initial data. In the absence of the magnetic potential $a$, the problem of recovering the electric potential $q$ on a compact Riemannian manifold was solved by Bellassoued and Dos Santos Ferreira [8].

The problem of determining the magnetic field by a local Dirichlet-to Neuman map was solved by Dos Santos Ferreira, Kenig, Sjöstrand and Uhlmann [5]. In [13], Tzou showed that the magnetic field depends stably on the Dirichlet-to Neuman map measured on any subboundary which is larger than half the boundary. In [4], Benjoud studied the inverse problem of recovering the magnetic field $d a$ and the electric potential $q$ from the knowledge of the Dirichlet-to-Neumann map. Assuming that the potentials are known in a neighborhood of the boundary, she proved a stability estimate with respect to arbitrary partial boundary observations. The key ingredient in the proof of all the above mentioned papers, is the construction of geometric optics solutions.

To our knowledge, there is a few results on the recovery of coefficients appearing in a Schrödinger equation, from a finite number of boundary measurements. By a method based essentially on an appropriate Carleman estimate, Baudouin and Puel [1] showed that the electric potential in the Shrödinger equation can be stably recovered from a single boundary measurement. In [7], Cristofol and Soccorsi proved a Lipschitz stability in recovering the magnetic field in the Schrödinger equation from a finite number of observations, measured on a subboundary for different choices of $u_{0}$. In the present paper, we improve the two above mentionned results by showing that the electric potential and the magnetic field can be stably and simultaneously recovered from a finite number of boundary observations of the solution. We stress out that the simultaneous identification of the magnetic field and the electric potential in the Schrödinger equation cannot be directly obtained from the results of [1] and [7], as the electric (resp. magnetic) potential is a two (resp. first) order perturbation of the laplacian. As a matter of fact, the method of derivation of the stability estimate given in Theorem 1.2 is different for the one of [7][Theorem 1.1], as second order time-derivatives of the solution only are used.

1.3. Well posedness of the magnetic Schrödinger equation and main results. Before stating our main result, we need first to justify the existence of a unique solution of (1.1). To this end, we introduce the space $\mathcal{H}_{1}(\Omega):=H_{0}^{1}(\Omega)$ equipped with the scalar product

$$
<(-\Delta+1)^{1 / 2} u,(-\Delta+1)^{1 / 2} v>_{L^{2}(\Omega)}, \quad \text { for any } u, v \in \mathcal{H}_{1}(\Omega),
$$

and denote by $\mathcal{H}_{2}:=H^{2}(\Omega) \cap H_{0}^{1}(\Omega)$ equipped with the scalar product

$$
<(-\Delta+1) u,(-\Delta+1) v>_{L^{2}(\Omega)} \quad \text { for any } u, v \in \mathcal{H}_{2}(\Omega) .
$$

Here and below $\left\langle\cdot, \cdot>_{L^{2}(\Omega)}\right.$ denotes the usual scalar product in $L^{2}(\Omega)$. Then, we have the following theorem: 
Theorem 1.1. Let $a \in A, q \in L^{\infty}(\Omega, \mathbb{R}), \chi \in \mathcal{C}^{3}(0, T ; \mathbb{R})$ and $\beta \in \mathcal{C}^{3}(0, T ; \mathbb{R})$. Then, for every $u_{0}$ satisfying $\Delta^{k} u_{0} \in \mathcal{H}_{2}, k=0,1,2$, and for any $f \in W^{2}\left(0, T ; \mathcal{H}_{2}(\Omega)\right)$, there exists a unique solution

$$
u \in \mathcal{C}^{2}\left(0, T ; \mathcal{H}_{2}\right) \cap \mathcal{C}^{3}\left(0, T ; \mathcal{H}_{0}\right)
$$

to the equation

$$
\begin{cases}\left(-i \partial_{t}+H_{a}(t)+\beta(t) q(x)\right) u(x, t)=f, & \text { in } Q=\Omega \times(0, T), \\ u\left(x, \frac{T}{2}\right)=u_{0}(x) & \text { in } \Omega .\end{cases}
$$

Moreover, there exists a constant $C>0$ such that

$$
\left\|\partial_{t}^{j} u(., t)\right\|_{\mathcal{H}_{1}(\Omega)} \leqslant C \sum_{k=0}^{j}\left\|\Delta^{k} u_{0}\right\|_{\mathcal{H}_{1}(\Omega)}, \quad j=0,1,2, \quad t \in(0, T) .
$$

Proof. Since $H_{a}(t)$ is a self adjoint operator in $L^{2}(\Omega)$, associated with the sesquilinear form

$$
u \mapsto\|(i \nabla+\chi(t) a)\|_{L^{2}(\Omega)^{n}}^{2}, \quad u \in H_{0}^{1}(\Omega),
$$

then, it holds true (see. e. g. [7]) that the domain of $H_{a}(t)$ is

$$
\mathcal{D}\left(H_{a}(t)\right)=H_{0}^{1}(\Omega) \cap H^{2}(\Omega) .
$$

Further, as $q \in L^{\infty}(\Omega)$ for all $t \in(0, T)$, we deduce from the Kato-Rellich Theorem that

$$
\mathcal{D}\left(H_{a}(t)+\beta(t) q\right)=\mathcal{D}\left(H_{a}(t)\right) .
$$

In view of [9][Section ], there exists a family of unitary operotors $(U(t, s))_{0 \leqslant s, t \leqslant T}$ in $\mathcal{H}_{0}$ satisfying the following statements:

(1) $U(s, s)=I d$, the identity mapping in $\mathcal{H}_{0}$,

(2) $U(t, s) \mathcal{D}\left(H_{a}(s)+\beta(s) q\right) \subset \mathcal{D}\left(H_{a}(t)+\beta(t) q\right), t, s \in[0, T]$.

(3) For all $\phi \in \mathcal{D}\left(H_{a}(s)+\beta(s) q\right)$, the mapping $t \mapsto U(t, s) x$, is continuously differentiable in $[0, T]$ and satisfies

$$
-i \partial_{t} U(t, s) \phi+\left(H_{a}(t)+\beta(t) q(x)\right) U(t, s) \phi=0,
$$

therefore, arguing as in [7][Section 2], we check that

$$
u(\cdot, t)=U\left(t, \frac{T}{2}\right) u_{0}+i \int_{\frac{T}{2}}^{t} U(t, s) f(s) d s,
$$

is solution to (1.3) and satisfies the estimate 1.4. We complete the proof of this theorem..

In order to express the main result of this paper, let us introduce some notations. Let us denote by $\mathcal{V}$, an arbitrary neighborhood of the boundary $\Gamma$. For $M>0$, and $\left(a_{0}, q_{0}\right) \in A \times L^{\infty}(\Omega)$, we define the admissible set of the unknown coefficients $a$ and $q$ :

$$
\mathcal{S}_{M}\left(a_{0}, q_{0}\right):=\left\{(a, q) \in A \times L^{\infty}(\Omega), \text { such that } a=a_{0}, \text { and } q=q_{0} \text { in } \mathcal{V}\right\} .
$$

Then, our main result can be stated as follows

Theorem 1.2. Let $M>0$, let $\chi$ and $\beta$ be as in Theorem 1.1 and satisfy $1.1 \quad$ Let $\left(a_{j}, q_{j}\right), j=1,2$ be in $\mathcal{S}_{M}\left(a_{0}, q_{0}\right)$, where $\left(a_{0}, q_{0}\right)$ are the same as above. Then, there exists $n+1$ initial conditions $u_{0, k}$, $k=0, \ldots, n$, such that we have

$$
\left\|a_{1}-a_{2}\right\|_{L^{2}(\Omega)}+\left\|q_{1}-q_{2}\right\|_{L^{2}(\Omega)} \leqslant C\left(\sum_{k=0}^{n}\left\|\partial_{\nu} \partial_{t}^{2} u_{1, k}-\partial_{\nu} \partial_{t}^{2} u_{2, k}\right\|_{L^{2}\left(0, T ; L^{2}\left(\Gamma^{+}\right)\right)}^{2}\right) .
$$


Here $C>0$ is a constant depending only on $\Omega, T, \chi$ and $\beta$ and $u_{j, k}, j=1,2$, is the solution of (1.1) where $u_{0, k}$ is substituted for $u_{0}$.

\subsection{Comments.}

- Notice that as in [9], we impose the initial condition $u\left(\cdot, \frac{T}{2}\right)=u_{0}$ in $\sqrt{1.1}$ ) at $t=\frac{T}{2}$ and not $t=0$. This allows us to "symmetrize" the solution $u$ to 1.1 around $t=\frac{T}{2}$, and consequently to apply the Carleman estimate of Proposition 2.1 to $u$ over $(0, T)$.

- The assumption $\nabla \cdot a=0$ is purely technical and does not restrict the generality of Theorem 1.2 . Indeed, it is well known that the magnetic potential is not meaningful in physics. The physical relevant quantity is the "two-form" $d a=\partial \wedge a$, which coïncides with the magnetic field curl $a$ when $n=3$. Actually, given the "magnetic field" $b$, we can always choose a divergence free $a$ such that we have $b=d a$. This amounts to substituting $a+\nabla \psi$ for $a$, where $\psi \in H^{1}(\Omega)$ is solution to the system

$$
\begin{cases}-\Delta \psi=\nabla \cdot a & \text { in } \Omega, \\ \psi=0 & \text { in } \partial \Omega\end{cases}
$$

- As in [7], we enforce homogeneous Dirichlet-boundary conditions to (1.1). These homogeneous Dirichlet conditions impose that $q$ be known in the vicinity $\mathcal{V}$ of the boundary $\partial \Omega$. Nevertheless, this condition can be removed upon selecting suitable non-homogeneous Dirichlet boundary conditions on $\partial \Omega$ as in [1].

- Similarly, we can remove the assumption that $a$ be known on $\mathcal{V}$ by selecting the initial conditions $u_{0, k}$, for $k=1, \ldots, n$, as in [7][Theorem 1.1]. Nevertheless, the set of initial conditions cannot be defined explicitly, so we rather stick with the formulation of Theorem 1.2 given in this paper. Nevertheless, in order to avoid the inadequate expense of the site of this article, we shall not go further into details in this matter.

- Evidently, it can be checked that if $a_{1}=a_{2}$ then, the electric potential can be Lipschitz stably retrieved from one boundary observation of the solution. This extends the result of Baudouin and Puel [1], to the case of a magnetic Laplacian.

Similarly, if $q_{1}=q_{2}$, we can determine the divergence free magnetic potential for $n$ boundary observations of the solution, which generalises the result of [7].

- It is worth mentionning that the stability estimate of Theorem 1.2 determines $n+1$ unknown functions $\left(a_{1}, \ldots, a_{n}\right)$ and $q$ from the knowledge of $n+1$ boundary observations over the time-space $(0, T)$.

- We stress out that the condition 1.1 imposed on the functions $\chi$ and $\beta$ are essential in order to solve the inverse problem under study in this work. Indeed they allow us to recover the information on $a$ and $q$ from the knowledge of the "initial" condition of the second order derivative of the linearized system associated with 1.1 (see the second line in 3.11).

1.5. Outline of the paper. The proof of Theorem 1.2 is based on the celebrated Bugkheim. Klibanov method [6] which is by means of a Carleman estimate designed for Schrödinger equations. In Section 2. we state the global Carleman estimate we use in the derivation of the stability result. Section 3 contains the proof of Theorem 1.2 . 


\section{Global Carleman estimate}

In this section, we recall the global Carleman inequality for vanishing solution on the boundary $\Sigma$, which can be found in [1][Section3]( see also [7] ). This estimate is the main tool needed for the derivation of Theorem 1.2.

Given the Schrödinger operator

$$
L:=i \partial_{t}+\Delta
$$

we define a function $\psi \in \mathcal{C}^{4}\left(\Omega, \mathbb{R}_{+}\right)$, satisfying the following conditions:

(i) $|\nabla \psi(x)| \geqslant \beta>0, \forall x \in \Omega$.

(ii) $\nabla \psi \cdot \nu<0$ for all $x \in \Gamma \backslash \Gamma^{+}$

(iii) $\exists \Lambda_{1}>0, \exists \varepsilon>0$ such that for all $\xi \in \mathbb{R}^{n}$, and for all $\lambda>\Lambda_{1}$, we have

$$
\lambda|\nabla \psi \cdot \xi|^{2}+D^{2} \psi(\xi, \bar{\xi}) \geqslant \varepsilon|\xi|^{2}
$$

where $D^{2} \psi=\left(\frac{\partial^{2} \psi}{\partial x_{i} \partial x_{j}}\right)_{1 \leqslant i, j \leqslant n}$ and $D^{2} \psi(\xi, \bar{\xi})$ denotes the $\mathbb{C}^{n}$-scalar product of $D^{2} \tilde{\psi} \xi$ with $\xi$.

Notice that there are actual functions $\psi$ verifying the above assumptions, such as $x \mapsto\left|x-x_{0}\right|^{2}$, for an arbitrary $x_{0} \in \mathbb{R}^{n} \backslash \bar{\Omega}$ and a subboundary $\Gamma^{+} \supset\left\{x \in \Gamma,\left(x-x_{0}\right) \cdot \nu \geqslant 0\right\}$. Furthermore, for $\lambda>0$ the following weight functions:

$$
\theta(x, t)=\frac{e^{\lambda \psi(x)}}{t(T-t)}, \quad \text { and } \quad \eta(x, t)=\frac{\alpha-e^{\lambda \psi(x)}}{t(T-t)},
$$

where $\alpha>\left\|e^{\lambda \psi}\right\|_{L^{\infty}(\Omega)}$. Finaly, we introduce the two operators $P_{1}$ and $P_{2}$ acting in $\mathcal{C}_{0}^{\infty}(Q)^{\prime}$, as follows:

$$
P_{1}:=i \partial_{t}+\Delta+s^{2}|\nabla \eta|^{2}, \quad \text { and } \quad P_{2}:=i s \partial_{t} \eta+2 s \nabla \eta \cdot \nabla+s(\Delta \eta),
$$

in such a way that $P_{1}+P_{2}=e^{-s \eta} L e^{s \eta}$. Then, we have the following result:

Proposition 2.1. Assume that $\psi$ and $\Gamma^{+}$satisfy the above conditions. Let $\eta$ and $\theta$ be as in (2.6), and let $P_{j}$, $j=1,2$ be defined by (2.7). Then, there are two constants $s_{0}>0$ and $C>0$, depending only on $T, \Omega$ and $\Gamma^{+}$, such that the estimate

$$
\begin{aligned}
& s\left\|e^{-s \eta} \nabla u\right\|_{L^{2}(Q)}^{2}+s^{3}\left\|e^{-s \eta} u\right\|_{L^{2}(Q)}^{2}+\sum_{j=1,2}\left\|P_{j} e^{-s \eta} u\right\|_{L^{2}(Q)}^{2} \\
& \quad \leqslant C\left(s\left\|e^{-s \eta} \theta^{1 / 2}\left(\partial_{\nu} \psi\right)^{1 / 2} \partial_{\nu} u\right\|_{L^{2}\left(\Sigma^{+}\right)}^{2}+\left\|e^{-s \eta} L u\right\|_{L^{2}(Q)}^{2}\right),
\end{aligned}
$$

holds for all $s \geqslant s_{0}$, and for any function $u \in L^{2}\left(0, T ; H_{0}^{1}(\Omega)\right)$ such that $L u \in L^{2}(Q)$ and $\partial_{\nu} u \in$ $L^{2}\left(0, T ; L^{2}\left(\Gamma^{+}\right)\right)$. Here $\Sigma_{T}^{+}$stands for $\Gamma^{+} \times(0, T)$.

\section{Stability estimate}

In this section, we derive the stability estimate for $a$ and $q$ appearing in the magnetic Schrödinger equation 1.1. of Theorem 1.2. Here and henceforth the symbol "'" stands for the differentiation with respect to $t$. 
3.1. Linearization. Let $u_{j}$, for $j=1,2$, be solutions to

$$
\begin{cases}\left(-i \partial_{t}+H_{a_{j}}(t)+\beta(t) q_{j}\right) u_{j}=0, & \text { in } Q \\ u_{j}\left(\cdot, \frac{T}{2}\right)=u_{0}(x), & \text { in } \Omega \\ u_{j}=0, & \text { on } \Sigma\end{cases}
$$

Then, $u=u_{1}-u_{2}$ is a solution to the following boundary value problem

$$
\begin{cases}\left(-i \partial_{t}+H_{a_{1}}+\beta(t) q_{1}\right) u=f, & \text { in } Q \\ u\left(\cdot, \frac{T}{2}\right)=0 & \text { in } \Omega \\ u=0 & \text { on } \Sigma\end{cases}
$$

where $f=\chi\left(a_{1}-a_{2}\right) \cdot\left(-2 i \nabla-\chi\left(a_{1}+a_{2}\right)\right) u_{2}-\beta\left(q_{1}-q_{2}\right) u_{2}$. By differentiating [3.9], we get

$$
\begin{cases}\left(-i \partial_{t}+H_{a_{1}}+\beta(t) q_{1}\right) v=g:=f^{\prime}-H_{a_{1}}^{\prime} u-\beta^{\prime} q_{1} u, & \text { in } Q, \\ v\left(\cdot, \frac{T}{2}\right)=0, & \text { in } \Omega, \\ v=0, & \text { on } \Sigma\end{cases}
$$

with $v=\partial_{t} u$. Thus, $w=\partial_{t} v$ is a solution to

$$
\begin{cases}\left(-i \partial_{t}+H_{a_{1}}(t)+\beta(t) q_{1}\right) w=h:=f^{\prime \prime}-2\left(H_{a_{1}}^{\prime}+\beta^{\prime} q_{1}\right) v-\left(H_{a_{1}}^{\prime \prime}+\beta^{\prime \prime} q_{1}\right) u, & \text { in } Q \\ w\left(\cdot, \frac{T}{2}\right)=2 \chi^{\prime}\left(\frac{T}{2}\right)\left(a_{1}-a_{2}\right)(x) \cdot \nabla u_{0}-i \beta^{\prime}\left(\frac{T}{2}\right)\left(q_{1}-q_{2}\right)(x) u_{0}, & \text { in } \Omega \\ w=0, & \text { on } \Sigma\end{cases}
$$

3.2. Preliminary estimates. We start by stating a powerful tool introduced by A. L. Bughkeim and M. V. Klibanov in [6].

Lemma 3.1. For $d \in\{1, \ldots, n\}$, let $\eta$ be given by (2.7). Then, there exists a positive constant $\kappa>0$, depending only on $T$, such that we have

$$
\int_{0}^{T} \int_{\Omega} e^{-2 s \eta(x, t)}\left|\int_{\frac{T}{2}}^{t} p(\xi, x) d \xi\right|^{2} d x d t \leqslant \frac{\kappa}{s}\left\|e^{-s / \eta} p\right\|_{L^{2}(Q)^{d}}
$$

for every $p \in L^{2}\left(Q_{T}\right)^{d}$.

We turn now to establishing the coming statement with the aid of Proposition 2.1 and the above lemma.

Lemma 3.2. There exists $s_{1}>0$ such that for any $s \geqslant s_{1}$, we have the following estimate

$$
\begin{aligned}
s^{3}\left\|e^{-s \eta} w\right\|_{L^{2}(Q)}^{2}+ & \left\|P_{1} e^{-s \eta} w\right\|_{L^{2}(Q)}^{2} \leqslant C\left(\left\|e^{-s \eta}\left(a_{1}-a_{2}\right)\right\|_{L^{2}(Q)^{n}}^{2}+\left\|e^{-s \eta}\left(q_{1}-q_{2}\right)\right\|_{L^{2}(Q)}^{2}\right. \\
& \left.+s\left\|e^{-s \eta} \theta^{1 / 2}\left(\partial_{\nu} \psi\right)^{1 / 2} \partial_{\nu} w\right\|_{L^{2}\left(\Sigma^{+}\right)}^{2}\right),
\end{aligned}
$$

where $C$ is a positive constant independent of $s$.

Proof. By applying Proposition 2.1 to the solution $w$, we find a constant $C>0$ such that

$$
\begin{aligned}
& s^{3}\left\|e^{-s \eta} w\right\|_{L^{2}(Q)}^{2}+\left\|P_{1} e^{-s \eta} w\right\|_{L^{2}(Q)}^{2}+s\left\|e^{-s \eta} \nabla w\right\|_{L^{2}(Q)}^{2} \\
& \quad \leqslant C\left(\left\|e^{-s \eta} L w\right\|_{L^{2}(Q)}^{2}+s\left\|e^{-s \eta} \theta^{1 / 2}\left(\partial_{\nu} \psi\right)^{1 / 2} \partial_{\nu} w\right\|_{L^{2}\left(\Sigma^{+}\right)}^{2}\right), \quad s>s_{0},
\end{aligned}
$$

where $L w(x, t)=\left(-h(x, t)+2 i \chi(t) a_{1}(x) \cdot \nabla+\chi^{2}(t) a_{1}^{2}(x)+\beta(t) q_{1}(x)\right) w(x, t)$. Here $h(x, t)$ is given by the following identity

$$
h(x, t)=-2\left(H_{a_{1}}^{\prime}+\beta^{\prime} q_{1}\right) v-\left(H_{a_{1}}^{\prime \prime}(t)+\beta^{\prime \prime}(t) q_{1}\right) u+f_{1}\left(q_{1}-q_{2}\right)(x)+f_{2}\left(a_{1}-a_{2}\right)(x),
$$


SIMULTANEOUS DETERMINATION OF THE MAGNETIC FIELD AND THE ELECTRIC POTENTIAL IN THE SCHRÖDINGER EQUATION where

$$
f_{1}(x, t)=\beta^{\prime \prime} u_{2}+2 \beta^{\prime} \partial_{t} u_{2}+\beta \partial_{t}^{2} u_{2},
$$

and

$$
\begin{gathered}
f_{2}(x, t)=\chi^{\prime \prime}\left(-2 i \nabla-\chi\left(a_{1}+a_{2}\right)-2 \chi^{\prime 2}\left(a_{1}+a_{2}\right)-\chi \chi^{\prime \prime}\left(a_{1}+a_{2}\right) u_{2}\right. \\
+2 \chi^{\prime}\left(-2 i \nabla-\chi\left(a_{1}+a_{2}\right)-2 \chi \chi^{\prime}\left(a_{1}+a_{2}\right)\right) \partial_{t} u_{2} \\
+\chi\left(-2 i \nabla-\chi\left(a_{1}-a_{2}\right)\right) \partial_{t}^{2} u_{2}
\end{gathered}
$$

In view of $\left[1.4\right.$, we have $f_{j} \in \mathcal{C}^{0}\left([0, T] ; L^{\infty}(\Omega)\right)$ for $j=1,2$. Moreover, it is easy to see that $H_{a_{1}}^{\prime}+\beta^{\prime} q_{1}$ and $H_{a_{1}}^{\prime \prime}+\beta^{\prime \prime} q_{1}$ are bounded operators from $L^{2}\left(0, T ; H^{1}(\Omega)\right)$ into $L^{2}(Q)$. Thus, there exists $C>0$, independent of $s$, such that we have

$$
\begin{gathered}
s^{3}\left\|e^{-s \eta} w\right\|_{L^{2}(Q)}^{2}+\left\|P_{1} e^{-s \eta} w\right\|_{L^{2}(Q)}^{2}+s\left\|e^{-s \eta} \nabla w\right\|_{L^{2}(Q)}^{2} \\
\leqslant C\left(\left\|e^{-s \eta}\left(a_{1}-a_{2}\right)\right\|_{L^{2}(Q)^{n}}^{2}+\left\|e^{-s \eta}\left(q_{1}-q_{2}\right)\right\|_{L^{2}(Q)}^{2}+s\left\|e^{-s \eta} \theta^{1 / 2}\left(\partial_{\nu} \psi\right)^{1 / 2} \partial_{\nu} w\right\|_{L^{2}\left(\Sigma^{+}\right)}^{2}\right. \\
\left.+\sum_{\rho=u, v, w}\left(\left\|e^{-s \eta} \rho\right\|_{L^{2}\left(Q_{T}\right)}^{2}+\left\|e^{-s \eta} \nabla \rho\right\|_{L^{2}(Q)}^{2}\right)\right) .
\end{gathered}
$$

Therefore, since $\nabla^{k} u(\cdot, t)=\int_{\frac{T}{2}}^{t} \nabla^{k} v(\cdot, \tau) d \tau$ and $\nabla^{k} v(\cdot, t)=\int_{\frac{T}{2}}^{t} \nabla^{k} w(\cdot, \tau) d \tau$, for $k=0,1$, we deduce from Lemma 3.1 that

$$
\begin{gathered}
s^{3}\left\|e^{-s \eta} w\right\|_{L^{2}(Q)}^{2}+\left\|P_{1} e^{-s \eta} w\right\|_{L^{2}(Q)}^{2}+s\left\|e^{-s \eta} \nabla w\right\|_{L^{2}(Q)}^{2} \\
\leqslant C\left(\left\|e^{-s \eta}\left(a_{1}-a_{2}\right)\right\|_{L^{2}(Q)^{n}}^{2}+\left\|e^{-s \eta}\left(q_{1}-q_{2}\right)\right\|_{L^{2}(Q)}^{2}+s\left\|e^{-s \eta} \theta^{1 / 2}\left(\partial_{\nu} \psi\right)^{1 / 2} \partial_{\nu} w\right\|_{L^{2}\left(\Sigma^{+}\right)}^{2}\right. \\
\left.+\left\|e^{-s \eta} w\right\|_{L^{2}\left(Q_{T}\right)}^{2}+\left\|e^{-s \eta} \nabla w\right\|_{L^{2}(Q)}\right),
\end{gathered}
$$

for any $s \geqslant s_{0}$. Thus, by taking $s$ sufficiently large, we obtain

$$
\begin{aligned}
s^{3}\left\|e^{-s \eta} w\right\|_{L^{2}(Q)}^{2}+ & \left\|P_{1} e^{-s \eta} w\right\|_{L^{2}(Q)}^{2} \leqslant C\left(\left\|e^{-s \eta}\left(a_{1}-a_{2}\right)\right\|_{L^{2}(Q)}^{2}+\left\|e^{-s \eta}\left(q_{1}-q_{2}\right)\right\|_{L^{2}(Q)}^{2}\right. \\
& \left.+s\left\|e^{-s \eta} \theta^{1 / 2}\left(\partial_{\nu} \psi\right)^{1 / 2} \partial_{\nu} w\right\|_{L^{2}\left(\Sigma^{+}\right)}^{2}\right) .
\end{aligned}
$$

This completes the proof of the Lemma.

3.3. Proof of Theorem 1.2. Let us now complete the stability estimate. Putting $\phi(x, t)=e^{-s \eta(x, t)} w(x, t)$ and using the fact that $\phi(x, 0)=0$, we get

$$
\left\|\phi\left(\cdot, \frac{T}{2}\right)\right\|_{L^{2}(\Omega)}^{2}=\int_{0}^{\frac{T}{2}} \int_{\Omega} \partial_{t}|\phi(x, t)|^{2} d x d t=2 \Re\left(\int_{0}^{\frac{T}{2}} \int_{\Omega} \partial_{t} \phi(x, t) \overline{\phi(x, t)} d x d t\right) .
$$

Hence, from the Green formula and (2.7) one can see that

$$
\begin{aligned}
\left\|\phi\left(\cdot, \frac{T}{2}\right)\right\|_{L^{2}(\Omega)}^{2} & =2 \Im\left(\int_{0}^{\frac{T}{2}} \int_{\Omega}\left(i \partial_{t}+\Delta+s^{2}|\nabla \eta(x, t)|^{2}\right) \phi(x, t) \overline{\phi(x, t)} d x d t\right) \\
& =2 \Im\left(\int_{0}^{\frac{T}{2}} \int_{\Omega} P_{1} \phi(x, t) \overline{\phi(x, t)} d x d t\right) .
\end{aligned}
$$


Therefore, we get from the Cauchy-Schwarz inequality that

$$
\begin{aligned}
\left\|\phi\left(\cdot, \frac{T}{2}\right)\right\|_{L^{2}(\Omega)}^{2} & \leqslant 2\left\|P_{1} \phi\right\|_{L^{2}(Q)}^{2}\|\phi\|_{L^{2}(Q)}^{2} \\
& \leqslant s^{-3 / 2}\left(s^{3}\left\|e^{-s \eta} w\right\|_{L^{2}(Q)}^{2}+\left\|P_{1} e^{-s \eta} w\right\|_{L^{2}(Q)}^{2}\right), \quad s>0 .
\end{aligned}
$$

Then, by Lemma 3.2, we obtain for all $s \geqslant s_{2}$

$$
\begin{aligned}
& \text { (3.16) }\left\|\phi\left(\cdot, \frac{T}{2}\right)\right\|_{L^{2}(\Omega)}^{2}=4 \chi^{\prime}\left(\frac{T}{2}\right)^{2}\left\|e^{-s \eta\left(\cdot, \frac{T}{2}\right)}\left(a_{1}-a_{2}\right) \cdot \nabla u_{0}\right\|_{L^{2}(\Omega)^{n}}^{2}+\beta^{\prime}\left(\frac{T}{2}\right)^{2}\left\|e^{-s \eta\left(\cdot, \frac{T}{2}\right)}\left(q_{1}-q_{2}\right) u_{0}\right\|_{L^{2}(\Omega)}^{2} \\
& \leqslant C s^{-3 / 2}\left(\left\|e^{-s \eta}\left(a_{1}-a_{2}\right)\right\|_{L^{2}(Q)^{n}}^{2}+\left\|e^{-s \eta}\left(q_{1}-q_{2}\right)\right\|_{L^{2}(Q)}^{2}+s\left\|e^{-s \eta} \theta^{1 / 2}\left(\partial_{\nu} \psi\right)^{1 / 2} \partial_{\nu} w\right\|_{L^{2}\left(\Sigma^{+}\right)}^{2}\right),
\end{aligned}
$$

Let us now choose the initial conditions $u_{0}$ as follows. Pick $\omega \subseteq \Omega$ such that $\omega \supset \Omega \backslash \mathcal{V}$. Then, we choose $u_{0} \in C_{0}^{6}(\Omega)$ such that $u_{0}(x)=1$ for any $x \in \omega$. Taking into account that $q_{1}-q_{2}$ and $a_{1}-a_{2}$ vanish in $\mathcal{V}$ and that $\eta\left(x, \frac{T}{2}\right) \leqslant \eta(x, t)$ for all $x \in \Omega$, we deduce from 3.16 that

$C_{1}\left\|e^{-s \eta\left(\cdot, \frac{T}{2}\right)}\left(q_{1}-q_{2}\right)\right\|_{L^{2}(\Omega)}^{2} \leqslant C s^{-3 / 2}\left(\left\|e^{-s \eta\left(\cdot, \frac{T}{2}\right)}\left(a_{1}-a_{2}\right)\right\|_{L^{2}(\Omega)^{n}}^{2}+\left\|e^{-s \eta\left(\cdot, \frac{T}{2}\right)}\left(q_{1}-q_{2}\right)\right\|_{L^{2}(\Omega)}^{2}+s\left\|\partial_{\nu} w\right\|_{L^{2}\left(\Sigma^{+}\right)}^{2}\right)$.

Here we used the fact that $\theta e^{-2 s \eta}$ and $\partial_{\nu} \psi$ are bounded on $\Sigma^{+}$. Next, we select $n$ initial conditions $u_{0, k} \in$ $C_{0}^{6}(\Omega)$, for $k=1, \ldots, n$, such that $u_{0, k}=x_{k}$ on $\omega$. Then, we infer from 3.16 in a similar way that (3.19)

$C_{2}\left\|e^{-s \eta\left(\cdot, \frac{T}{2}\right)}\left(a_{1}-a_{2}\right)_{k}\right\|_{L^{2}(\Omega)}^{2} \leqslant C s^{-3 / 2}\left(\left\|e^{-s \eta\left(\cdot, \frac{T}{2}\right)}\left(a_{1}-a_{2}\right)\right\|_{L^{2}(\Omega)^{n}}^{2}+\left\|e^{-s \eta\left(\cdot, \frac{T}{2}\right)}\left(q_{1}-q_{2}\right)\right\|_{L^{2}(\Omega)}^{2}+s\left\|\partial_{\nu} w\right\|_{L^{2}\left(\Sigma^{+}\right)}^{2}\right)$,

where $\left(a_{1}-a_{2}\right)_{k}$ denotes the $k^{\text {th }}$ component of $a_{1}-a_{2}$. Summing up (3.18) with 3.19 ) for $k=1, . ., n$, we get that

$$
\begin{gathered}
C_{1}\left\|e^{-s \eta\left(\cdot, \frac{T}{2}\right)}\left(q_{1}-q_{2}\right)\right\|_{L^{2}(\Omega)}^{2}+C_{2} \sum_{j=1}^{n}\left\|e^{-s \eta\left(\cdot, \frac{T}{2}\right)}\left(a_{1}-a_{2}\right)_{j}\right\|_{L^{2}(\Omega)}^{2} \\
\leqslant C(n+1) s^{-3 / 2}\left(\left\|e^{-s \eta(\cdot, 0)}\left(a_{1}-a_{2}\right)\right\|_{L^{2}(\Omega)^{n}}^{2}+\left\|e^{-s \eta\left(\cdot, \frac{T}{2}\right)}\left(q_{1}-q_{2}\right)\right\|_{L^{2}(\Omega)}^{2}+s\left\|\partial_{\nu} w\right\|_{L^{2}\left(\Sigma^{+}\right)}^{2}\right), \quad s \geqslant s_{2}
\end{gathered}
$$

Thus, there exists $s_{3}>0$ such that for $s \geqslant s_{3}$, we have

$$
\left\|e^{-s \eta\left(\cdot, \frac{T}{2}\right)}\left(q_{1}-q_{2}\right)\right\|_{L^{2}(\Omega)}^{2}+\left\|e^{-s \eta\left(\cdot, \frac{T}{2}\right)}\left(a_{1}-a_{2}\right)\right\|_{L^{2}(\Omega)^{n}}^{2} \leqslant C s^{-1 / 2}\left\|\partial_{\nu} \partial_{t}^{2}\left(u_{1}-u_{2}\right)\right\|_{L^{2}\left(\Sigma^{+}\right)}^{2} .
$$

Finally, from the inequality $e^{-s \eta\left(\cdot, \frac{T}{2}\right)} \geqslant e^{-4 s \frac{\alpha-1}{T^{2}}}>0$, we get the desired result.

\section{REFERENCES}

[1] L. Baudouin, Jean-Pierre Puel Uniqueness and stability in an inverse problem for the Schrödinger equation,

[2] M. Bellassoued, Stable determination of coefficients in the dynamical Shrödinger equation in a magnetic field, arXiv:1510.04247v1.

[3] M. Bellassoued, M. Choulli, Stability estimate for an inverse problem for the magnetic Schödinger equation from the Dirichlet-to-Neumann map, Journal of Functional Analysis, 258, 1 (2010), 161-195.

[4] H. Ben Joud, Stability estimate for an inverse problem for the Schrödinger equation in a magnetic field from partial boundary meausurements, Inverse Problems (2009).

[5] D. Dos Santos Ferreira, C. E. Kenig, J. Sjöstrand, G. Uhlmann, Determining a magnetic Schrödinger operator from partial Cauchy data, Comm. Math. Phys. 2 (2007), 467-488.

[6] A. L. Bukhgeim, M. V. Klibanov, Uniqueness in the large of a class of multidimensional inverse problems, Soviet Math. Dokl. 17 (1981), 244-247.

[7] M. Cristofol, E. Soccorsi, Stability estimate in an inverse problem for non autonomous magnetic Schrödinger equations 
SIMULTANEOUS DETERMINATION OF THE MAGNETIC FIELD AND THE ELECTRIC POTENTIAL IN THE SCHRÖDINGER EQUATION

[8] M. Bellassoued, D. Dos Santos Ferreira, Stable determination of coefficients in the dynamical anisotropic Schrödinger equation from the Dirichlet-to-Neumann map, Inverse Problems 26 (2010) 125010 (30pp).

[9] S. Liu, R. Triggiani, Global uniqueness in determining electric potentials for a system of strongly coupled Schrödinger equations with magnetic potential terms

[10] G. Nakamura, Z. Sun, G. Uhlmann, Global identifiability for an inverse problem, Math. Ann. 303 (1995), $377-388$.

[11] G. Eskin, Inverse problems for the Schödinger equations with time-dependent electromagnetic potentials and the AharonovBohn effect, J. Math. Phys. 49 (2008), no.2, 022105, 18 pp. 11, 1737-1758.

[12] Z. Sun, An inverse boundary value problem for the Schrödinger operator with vector potentials, Trans. Amer. Math. Soc, Vol. 338. No. 2, (1992), 953-969.

[13] L. Tzou, Stability estimates for coefficients of magnetic Shrödinger equation from full and partial boundary measurements, Comm. Partial Differential Equations, 11 (2008), 1911-1952. 